\title{
Road Obstacle Detection Based on Randomized Hough Transform
}

\author{
Jing LV \\ College of Information Engineering, \\ Capital Normal University \\ Beijing, China \\ lvjing1990911@sina.com
}

\author{
Yuanyuan Shang \\ College of Information Engineering, \\ Capital Normal University \\ Beijing, China \\ syy@bao.ac.cn
}

\author{
Hui Ding \\ College of Information Engineering, \\ Capital Normal University \\ Beijing, China \\ dinghui@ie.cnu.edu.cn
}

\begin{abstract}
Road obstacle detection is the core of pedestrian safety. As an effective image object detection method, Hough transform can detect straight lines, circles, ellipses, parabolas, and many other analytical graphics. This paper mainly detects bar deceleration strips and round well covers, owing to obvious linear features. Therefore, randomized Hough transform is applied to detect roadblocks to ensure pedestrian safety, improving the detection efficiency compared to the classical Hough transform in this article.
\end{abstract}

Keywords- hough transform; randomized hough transform; road obstacle detection

\section{INTRODUCTION}

As the transportation safety problem has drawed increasing attention, the Road Obstacle Detection, an important aspect of intelligent transportation system, has gradually becoming a popular topic in domestic and international academic research ${ }^{[1]}$. At present, algorithms and implementation methods about the road obstacle detection include technology based on computer vision ${ }^{[2]}$, laser $\operatorname{radar}^{[3]}$, neural network, and so on. However, most of these researches take intelligent vehicle or robot ${ }^{[4]}$ as obstacle detection platform and choose traffic lane line or indoor obstacles $^{[5]}$ as research object. The road obstacle detection in this paper focus on pedestrian safety, the key factors of transportation safety. This paper can be applied to detection about raised roadblocks such as bar deceleration strips and round well covers.

Among current Image-based roadblocks detection methods, the most basic method is based on image feature, which extract color, edge and texture features as the road obstacle detection information ${ }^{[6]}$. But in case of fuzzy edge and impacts of light, those methods can not accurately extract edge information of obstacles, and are not targeted to the detection of bar deceleration strips and round well covers.

Road deceleration strips and round well covers present obvious linear features, as shown in the Fig.1, so road obstacle detection based on the linear features can be applied, and Hough transform is in common use ${ }^{[7]}$. Therefore, this paper uses an obstacle detection method based on Hough Transform.

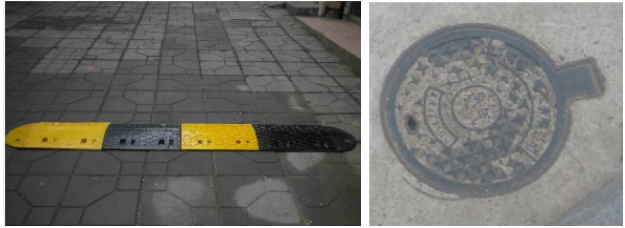

Figure 1. Bar deceleration strips and Round well covers.

\section{RANDOMIZED HOUGH TRANSFORM ALGORITHM}

\section{A. Randomized Hough Transform}

The Hough transform has advantages such as its algorithm has high calculation stability and has good antinoise performance. The disadvantage of Hough transform is that the calculation amount and storage space consume too much ${ }^{[8]}$. However, with the deepening of the research about Hough transform, a series of improved algorithm which follow the direction in reducing the time and space complexity have been put forward. $\mathrm{Xu}$ et al. proposed Randomized Hough Transform $(\mathrm{RHT})^{[9]}$, which uses a many-to-one converging mapping from the image space to the parameter space ${ }^{[10]}$, as shown in the Fig.2 (a), to replace the one-to-many diverging mapping applied in the classic Hough transformation, as shown in Fig.2 (b). It avoids a large storage requirement and low speed for real applications.

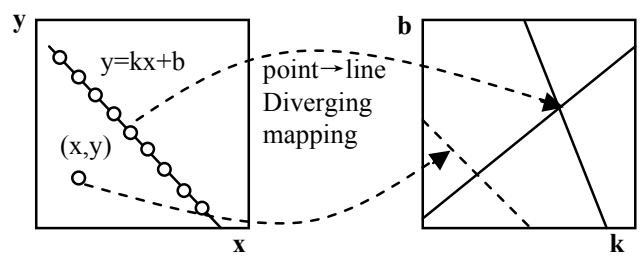

(a)

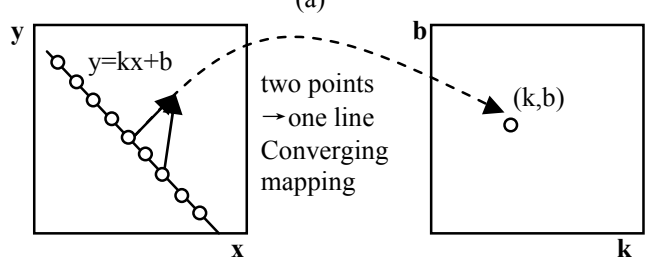

(b)

Figure 2. From Hough transform to Randomized Hough transform.

The basic idea of Hough transform (HT) is to implement a voting procedure for all potential curves in the image, and at the termination of the algorithm curves that do exist in the 
image will have relatively high voting scores. In comparison with HT, RHT takes advantage of the fact that some analytical curves can be fully determined by a certain number of points on the curve. For example, a straight line can be determined by two points, and an ellipse (or a circle) can be determined by three points.

This paper takes regular shape roadblocks as research emphasis. For obstacles such as road bar deceleration strips and round well covers, their contour points must exist in the same edge curve. So this paper uses road obstacle detection method based on Randomized Hough Transform aiming at independent edge curve.

\section{B. Lines detection using RHT}

In general, the contour image of the bar deceleration strip is approximately a straight line. So it is an effective method to detect deceleration strips through line detection base on RHT.

Therefore line detection using RHT is based on the fact that a single parameter space point can be determined uniquely with a pair of points from the original picture which can be chosen randomly from the edge image. As shown in the linear polar equation:

$$
\rho=x \cos \theta+y \sin \theta .
$$

Equation(1) denotes the coordinates of the points on the straight line. $\rho$ denotes the normal distance from the image origin to the line, and $\theta$ denotes the angle the normal line makes with $\mathrm{x}$-axis.

Once the score of a straight line by cumulative voting exceeds the threshold, it is determined as existing in the image (in other words, this line is detected), and should be removed from the image and accumulator array so that the algorithm can detect other straight lines faster. The algorithm terminates when the number of iterations reaches a maximum limit or all the lines have been detected. And RHT algorithm dynamics list structure, which improves computing speed and reduces storage space. RHT algorithm can be summarized as follows ${ }^{[11]}$.

1) Construct the set of points $D$ using all edge points of the image, and initialize the parameter space $P$ (save two parameters and accumulated value for the line to be detected) and the number of loops $k$.

2) Randomly selected two points from the set $D$.

3) Calculate the parameter point of the straight line determined by two points through the linear equation.

4) Looking parameter points within the allowable error in the parameter space $P$. And the accumulator plus 1 , while the number of loops plus 1 .

5) If the accumulator value or the number of loops exceeds a preset threshold, the corresponding parameter point is the parameter of the detected line. If corresponding points of this parameter is greater than the minimum number of points to determine a straight line, move the points of the line from the point set $D$ and reset $P$; otherwise, return step 2 .

The core idea of line detection using RHT is to reduce the calculation amount using random sampling in the image space, which does not vote for all pixels, aiming at avoid one-to-many mapping applied in the classic Hough transformation.

\section{Circles detection using RHT}

The basic approach of Circle detection using Hough Transform is to extract the parameters distributing at the target circle and the characteristics value of points to detect the circle. The general circle equation ${ }^{[12]}$ for the X-Y plane is:

$$
(x-a)^{2}+(y-b)^{2}=r^{2} .
$$

Thus it can be seen that determining a circle needs at least three elements: radius of the circle, Circle coordinates $\mathrm{x}$ and y-axis.

Similar with the principle of line detection, circle detection using RHT also starts by randomly selecting three points on the circles. And then update the accumulator array and corresponding scores. With the circle parameters determined from previous stage, output the circles with scores higher than some predefined threshold. Simultaneously, the maximum number of loops of allowable random sampling bases on the number of circles to be detected, which can avoid the excessive invalid detection.

\section{EXPERIMENTAL RESULTS AND ANALYSIS}

\section{A. Simple road obstacle detection methods}

First, a few simple image-based roadblock detection methods apply to experiments. Such as the adaptive thresholding method based on the color characteristics and the detection method based on edge features. The obstacle detection results as shown in Fig.3:
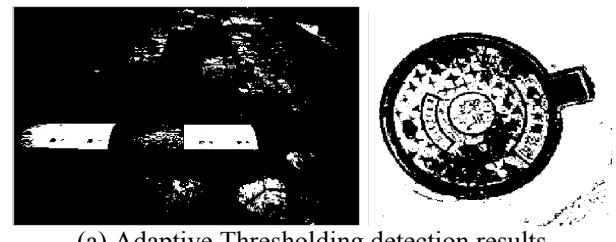

(a) Adaptive Thresholding detection results

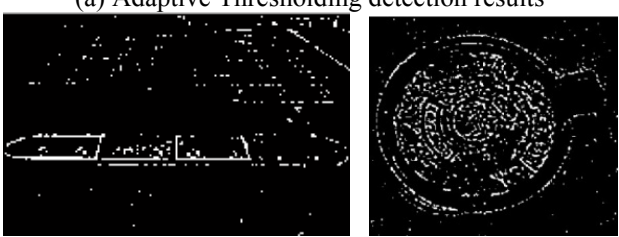

(b) Edge detection results

Figure 3. Simple road obstacle detection methods.

As can be seen from the experimental results, the results of simple detection methods can not accurately extract information of obstacles owing to more interference. More importantly, it can't provide stronger pertinence for obstacles such as road bar deceleration strips and round well covers.

\section{B. Road obstacle detection using RHT}

Thus in this paper, RHT algorithm directs to detect linear feature of obstacles, based on MATLAB, processing of color 
images. The road bar deceleration strips images are $640 * 480$ and round well covers images are $480 * 480$. The input of RHT algorithm is binary edge image, after the preprocessing steps, such as gray, filtering, binarization, edge detection and so on.

One of the major road obstacles categories, bar deceleration strips, has significant linear features, as shown in the left part of Fig.1. At the same time, another major road obstacles class is round well covers, especially prominent one, which is also a safety hazard for pedestrians and vehicles, as shown in the right part of Fig. 1 .

After the image pre-processing operations, road obstacles detection using RHT algorithm, whose input image is the binary edge image as shown in Fig.3 (b), is achievable by detecting lines and circles in the original image. The example detection results are shown in Fig.4.
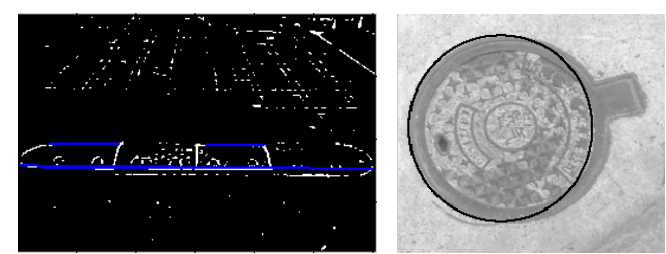

Figure 4. Road obstacle detection using RHT

This experiment tested 100 images of each obstacle categories. The detection accuracy and the average running time are shown in Tab.1.

TABLE I. EXPERIMENT RESULTS

\begin{tabular}{|c|c|c|}
\hline $\begin{array}{c}\text { Evaluation } \\
\text { Deteetion } \\
\text { type }\end{array}$ & $\begin{array}{c}\text { Detection } \\
\text { Accuracy }\end{array}$ & $\begin{array}{c}\text { Average running } \\
\text { time(s) (Raise rates } \\
\text { compare with HT) }\end{array}$ \\
\hline $\begin{array}{c}\text { bar deceleration strips } \\
\text { detection }\end{array}$ & $89 \%$ & $1.0768(63.6 \%)$ \\
\hline $\begin{array}{c}\text { round well covers } \\
\text { detection }\end{array}$ & $93 \%$ & $1.3733(83.3 \%)$ \\
\hline
\end{tabular}

\section{Experimental analysis}

Experimental results show that it is a very effective application that the method of line detection and circle detection using RHT is applied to road obstacles detection, including bar deceleration strips detection and round well covers detection. It can more accurately detect the position of the road obstacles, reflecting the Randomized Hough Transform has a unique capability of geometric feature detection, which provides an effective and highly targeted way for road obstacle detection and also provides a layer of pedestrian safety.

\section{CONCLUSION}

For the detection of pedestrian security, bar deceleration strips and round well covers are the main obstacle categories.
According to line detection and circle detection using RHT, this article devotes to detect and extract these primitives, owing to these roadblocks' line and circle features, applying to the detection of roadblocks. Hough transform has a strong anti-jamming capability, clear geometric analytic advantages and easy to implement. Due to strong fault tolerance and robustness of lines and circles detection, randomized Hough transform, which further improves the computing speed of the algorithm, reduces the memory requirements and computational needs, can effectively detect obstacles on the road in this paper.

\section{ACKNOWLEDGMENT}

This work was supported by National Natural Science Foundation of China (No. 11178017, 61373090, 61303104 and 61203238). And it was supported by Beijing Natural Science Foundation of China (4132014).

\section{REFERENCES}

[1] Zebbara KHALID, EL Ansari MOHAMED, Mazoul ABDENBI, "Stereo vision-based road obstacles detection," Intelligent Systems: Theories and Applications(SITA), 2013 8th IEEE Conference, Page(s):1-6.

[2] Yu-Chen Lin, Che-Tsung Lin, Wei-Cheng Liu, Long-Tai Chen, "A Vision-Based Obstacle Detection System for Parking Assistance," Industrial Electronics and Applications(ICIEA),2013 8th IEEE Conference: $1627-1630$.

[3] Inwook Shim, Dong-Geol Choi, Seunghak Shin, In So Kweon, "Multi Lidar System for fast obstacle detection," 2012 9th International Conference on Ubiquitous Robots and Ambient Intelligence (URAI):557-558.

[4] Daniel Maier, Maren Bennewitz, Cyrill Stachniss, "Self-supervised Obstacle Detection for Humanoid Navigation Using Monocular Vision and Sparse Laser Data," IEEE International Conference on Robotics and Automation, 2011.:1263-1269.

[5] Liu Hong, Wang Zhe, Wang Xiangdong, et al. Adaptive Scene Segmentation and Obstacle Detection for the Blind[J]. Journal of Computer-Aided Desigh \& Computer Graphics, 2013, 25(12): 18181825(in Chinese).

[6] LIN Chuan, SONG Wei-qi, QIN Jin-fei. Improved Method of Obstacle Detection Based on V- disparity[J]. Science Technology and Engineering,2014,14(1):86-90(in Chinese).

[7] D.H. Ballard. Generalizing the Hough Transform to Detect Arbitrary Shapes[J]. Pattern Recognition, 1981, 13(2):111-122.

[8] Lei XU, Erkki OJA, and Pekka Kultanena, "A new curve detection method: Randomized Hough Transform (RHT)," Pattern Recognition Letters, (11):331-338, 1990.

[9] XU L, OJA E, "Randomized Hough transform (RHT): basic mechanisms, algorithms, and computational complexities," CVGIPImage Understanding, 1993, 57(2):131-154.

[10] Lei Xu, Erkki Oja, "Randomized Hough Transform," Encyclopedia of Artificial Intelligence (3 Volumes), 2009:1354-1361.

[11] S. Inverso, "Ellipse Detection Using Randomized Hough Transform," www.saminverso.com/res/vision/EllipseDetection.pdf, September 12, 2006.

[12] McLaughlin and Robert, "Randomized Hough Transform: Improved Ellipse Detection with Comparison,” Technical Report JP98-01, 1998 